\title{
LSA System Development with Sensing for Rapidly Deployable LTE Network
}

\author{
Kalle Lähetkangas ${ }^{1}[0000-0001-7381-8549]$, Harri Posti ${ }^{1}$, Harri \\ Saarnisaari ${ }^{10000-0001-9857-3234]}$, and Ari Hulkkonen ${ }^{2}$ \\ 1 Centre for Wireless Communications, FI-90014 University of Oulu, Finland \\ \{Kalle.Lahetkangas, Harri.Posti, Harri.Saarnisaari\}@oulu.fi \\ 2 Bittium Wireless Ltd.,Ritaharjuntie 1, 90590 Oulu, Finland \\ Ari.Hulkkonen@bittium.com
}

\begin{abstract}
Public safety users require radio spectrum for their communication systems. In this study, sensors are proposed as a backup spectrum information source in a rapidly deployed public safety long term evolution (LTE) communication network with licensed shared access (LSA) system. While the LSA system has been well developed, the drawback measures have not been thoroughly investigated from the application point of view. Herein, a collaborative sensing method is suggested for detecting an incumbent spectrum user and for establishing a protection zone around it. Furthermore, methods are developed for combining information from sensors and from an LSA system in a rapidly deployable public safety LTE network. The information from the sensors can be used for verifying incumbent protection and also for finding available spectrum in critical scenarios. The proposed methods give wider spectrum knowledge than just by using repository information or local sensor information.
\end{abstract}

Keywords: Sharing arrangement · Commercial LTE network · dynamic spectrum access.

\section{Introduction}

Public safety (PS) actors today utilize closed communication networks, such as TETRA [11], as their primary communication systems. The advances in commercial networks have made them an attractive option for the PS actors who also want to benefit from the efficient technologies. The current standards for the long term evolution (LTE) networks are planned considering PS users as priority users [5-8]. This enables the commercial network providers to offer their services also to the PS actors, such as border control, police, first responders and military, whose effective communication capabilities are vital for the society. Moreover, the commercial LTE equipments are relatively inexpensive and the infrastructure is readily available.

While commercial networks have advantages over the traditional closed PS networks, the PS actors can not yet completely rely their communication over 
the commercial networks. This is because the commercial networks might not be built in every required location and because the full functionality of the community infrastructure might not be available in the PS operations due to electrical breaks or infrastructure damages. A solution to counter these uncertainties is a local rapidly deployable LTE network [16] which can be carried with the PS actors to the point of action. In this network the PS actors have local and full control for guaranteed services.

These deployable networks require radio spectrum. The spectrum may be owned by the PS users, but that is inefficient use of scarce radio spectrum since most of the time the PS spectrum is unused. Another way is spectrum sharing. The spectrum sharing can be enabled with a political decision. Alternatively, the commercial network operators can have monetary incentives to offer complete communication solutions including secondary spectrum rights for their PS customers. Note that the shared spectrum usage should not generally disturb the commercial networks. This is true especially when the commercial networks also serve priority users such as public safety.

One way of obtaining and sharing spectrum with the commercial systems interference free is licensed shared access (LSA) [3,9,10]. Another spectrum sharing method is called spectrum access system, which is designed for centrally sharing the $3.5 \mathrm{GHz}$ band in the United States $[21,25,20]$.

This work concentrates in LSA, where the secondary spectrum user requests the spectrum from a central spectrum repository via internet. An overview of spectrum sharing principles with LSA for PS from the point of spectrum ownership has been given in [15].

However, the spectrum sharing system for rapidly deployed public safety networks must operate also in some problematic situations. One is prolonged internet connection loss that denies access to the repository. Moreover, the information in the repository might not be valid. This is for example a crisis situation when the primary spectrum users such as commercial networks are down. For these situations, spectrum sensing to detect vacant spectrum is needed. Note, that the fall-back measures in LSA specifications [10] are presented only on a high level, and do not include sensing.

Spectrum sensing has been extensively studied in wireless communications $[26,13]$ and the sensing methods include, for example, energy detector $[2,14,17]$, known signal correlation, cyclostationary, and radio identification based sensing methods. The spectrum sensing dimensions are frequency, space, time, power, directional information and polarization.

Sensor networks have been proposed to be used together with the LSA system in [19] to map the radio environment to a central data base together with the LSA repository. In that work, collaborative use of sensor networks enables efficient spectrum sharing between multiple LSA licensees. Spectrum sensing can also be done collaboratively. The collaborative sensing has been surveyed in $[1,22,24]$. Furthermore, multiple sensors can be used to mitigate the effect of shadowing and fading by sharing their information [12, 18, 23]. 
This work utilizes the already existing sensing and detecting methods in a new kind of manner and do not develop another decision making algorithm or update a central data base. Our point of view is to utilize additional available sensors in conjunction of LSA system. The proposed methods are planned to be used as a simple backup system for spectrum information on PS network utilizing LSA when these types of centralized repository systems might not be functional. For the first time we have a decision method for LSA to select the allowed resources with contradicting spectrum information sources. More specifically, when the repositories are fully functional and the commercial networks are working, the repository information is verified with sensors. Then, when the LSA system has problems, the sensors are utilized for obtaining the spectrum information.

We take the spatial perspective for the sensors. More specifically, this work utilizes distances from which a sensor can detect incumbents. A collaborative sensing method is proposed for ensuring the available spectrum when there are multiple sensors available. Other collaborative methods probabilistically increase the detection probability by sensing with multiple sensors. Compared to these methods our method obtains a higher detection probability by simply considering smaller spatial areas that are reliably detected by a single detector. Furthermore, by having multiple sensors at different locations the reliably detected area broadens.

The paper is organized as follows. Section 2 presents the network model. Section 3 introduces a collaborative sensing method for locating the possible incumbent and describes how to find a protection zone around it. Section 4 describes how the sensors and LSA repository can be used together to allow spectrum utilization. Here, the spectrum use agreements between the incumbent and the LSA licensee, i.e. sharing arrangements, determine wether the use is allowed or not. Section 5 describes how to control false and miss detections. Section 6 describes the practical use of the sensors, for when the LTE base stations of public safety can disturb with the sensing. Section 7 is the conclusion.

\section{System model}

This work concentrates on a deployable closed PS Long Term Evolution (LTE) network, that acts as a secondary spectrum licensee in an LSA system. Fig. 1 presents the network components and their connections. The left side of the figure is the considered deployable network. It is used for PS communication when the commercial network might be unavailable or drastically limited. The LTE access points use frequency division duplex. The LSA repository offers available spectrum information for the LTE access points via an Internet Protocol (IP) network connection. ${ }^{3}$ The radio heads provide more spectrum information and are controlled with a sensor manager application. The distributed LSA

\footnotetext{
3 The commercial networks can be connected to this system for example with multiradio access technologies such as a high antenna gain multioperator $3 \mathrm{G} / 4 \mathrm{G} / \mathrm{LTE}$ router lifted high above the ground level.
} 
controllers use the channel information from the repository and from the radio heads. The controllers control the use of carrier frequency of the LTE access points and synchronize their spectrum information. The light EPC before every access point is a light version of evolved packet core. It offers the necessary services for connecting the access points to the backhaul network and enables a connection between the LTE access points. The backhaul is formed with a private IP network, that can be either wired or wireless [4]. In our scenarios the private IP backhaul network is considered to be highly robust and self configuring. Note, that the number of access points, radio heads and routers can vary. This type of a network can be built for example with towable lifts or crane cars, that are equipped with the necessary equipment.

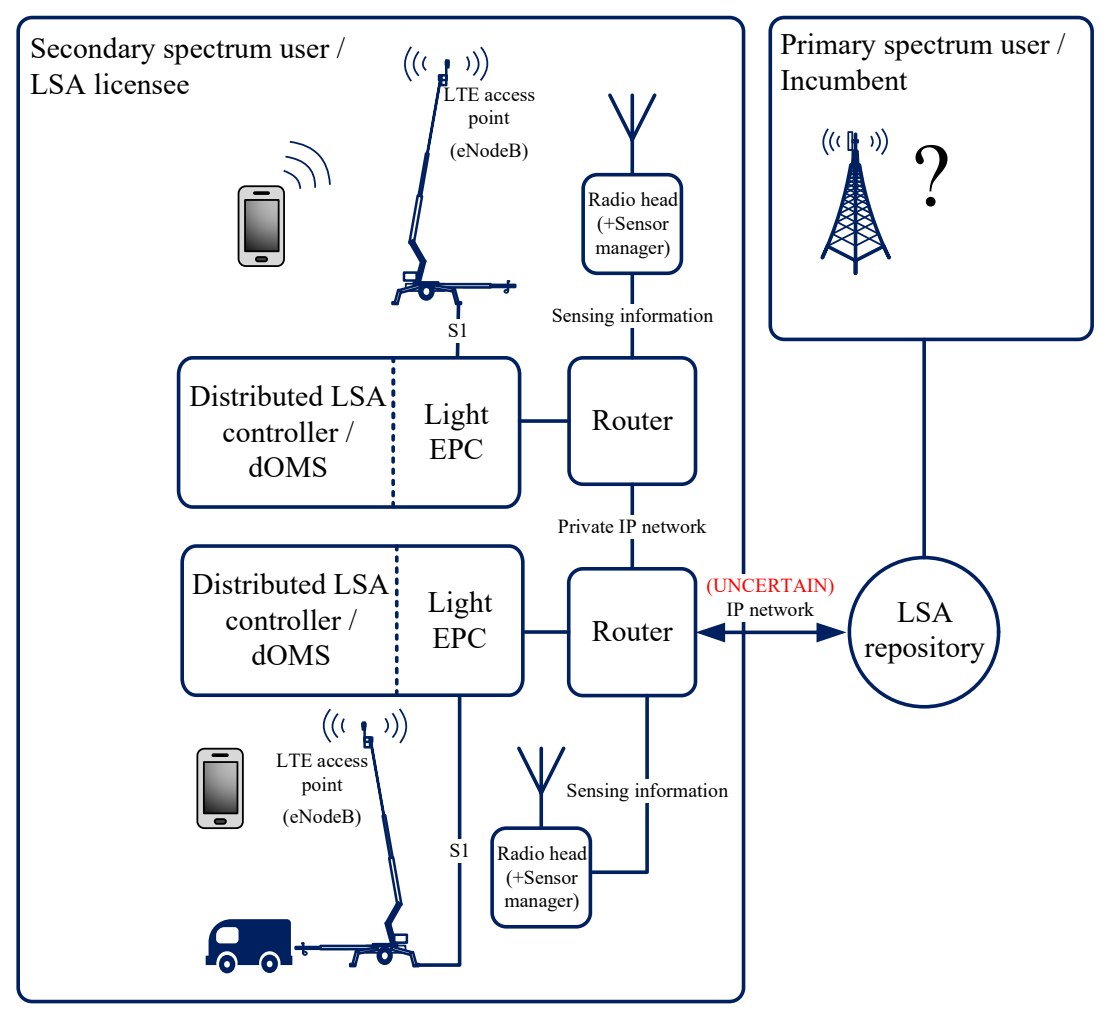

Fig. 1. LSA system for rapidly deployed LSA licensee network with LTE access points and radio heads for sensing. The primary spectrum users might be present, for which the sensing is used.

In order to analyze incumbent free areas with multiple sensors, the detection distances of a sensor in various conditions have to be defined. The distances depend on the specific sensing method, on transmitted signal power, on antenna 
gains, on attenuation, on random noise, on shadowing and this is not in the scope of this paper.

\section{Collaborative sensing method}

In this section, collaborative sensing is introduced for multiple sensors to sense if the channel is free or not and to calculate a protection zone for a possible incumbent user. The basic principle for collaboratively sensing the channel free is shown Figs. 2 and 3.

First, circles with radius $d_{\mathrm{f}}$ are drawn around the sensors that do not detect the incumbent. A distance $d_{\mathrm{f}}$ denotes the radius that is likely free of transmitters, given that there is no detection. Second, the union of these circles is taken. It is seen in Fig. 3 inside the solid thin red line. This area is free of the incumbent transmitter. Finally, the area within distance $d_{\text {inc }}$ is removed from the boundary of the union. A distance $d_{\text {inc }}$ is the maximum distance between the incumbent transmitter and the incumbent receiver. Removing this area, mitigates the hidden node problem. The remaining area is free of incumbent receivers. This area is seen in Fig. 3 inside the solid and rounded thick green line. Its complement is the protection zone, which is the area within which the incumbent receivers should not be subject to harmful interference caused by PS. The above method

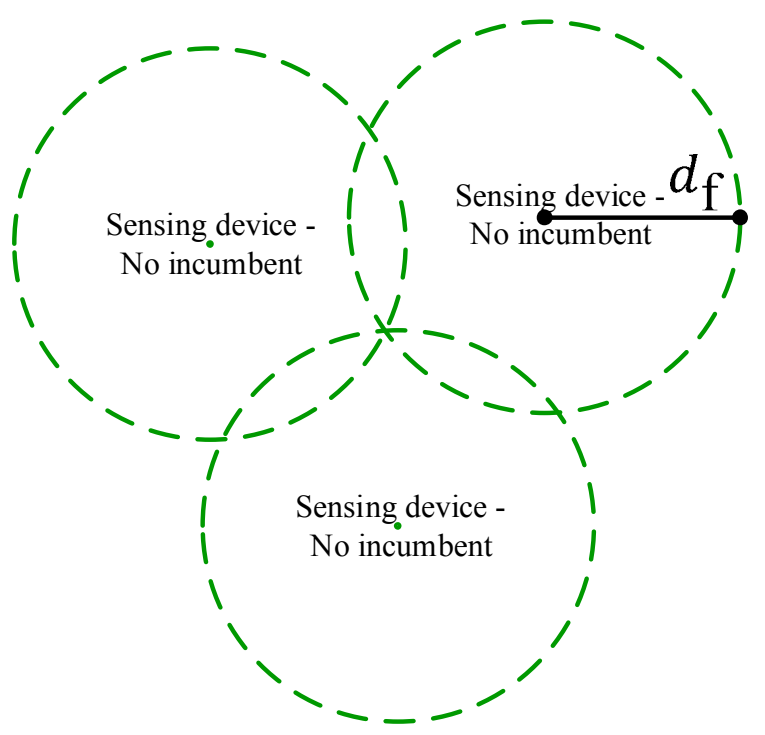

Fig. 2. Three sensors with no detection.

can also be utilized when there are multiple incumbent transmitters, but no detection. Note that multiple transmitters increase the distance from which the 


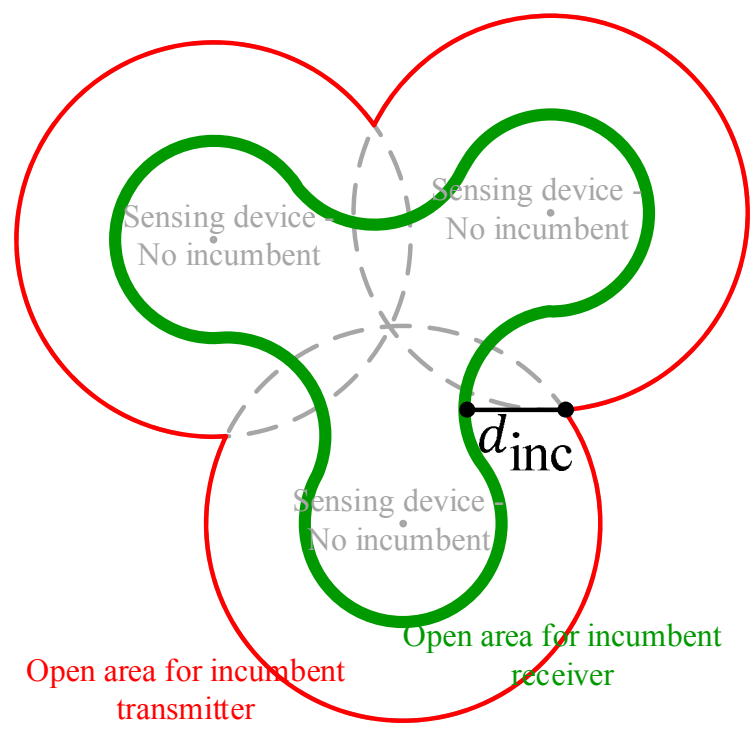

Fig. 3. Three sensors do not sense the incumbent. LSA controllers caluclate an incumbent free zone for a possible incumbent, whose receiver is within distance $d_{\text {inc }}$ from the transmitter.

incumbent can be detected. Thus, the transmitter free distance calculated for a single incumbent $d_{\mathrm{f}}$ is free also in a multiple transmitter scenario.

If there is only one incumbent transmitter, multiple sensors can be used to roughly locate it. The basic principle can be seen from Fig. 4. In this example, two sensors have sensed the incumbent and one sensor has not. First, circles with radius $d_{\mathrm{t}}$ are drawn around the sensors that detect the incumbent and an intersection between these circles is taken. A distance $d_{\mathrm{t}}$ denotes the radius within the detected LTE transmitter is with a close to one probability. Second, circles with radius $d_{\mathrm{f}}$ are drawn around all the sensors that do not detect the incumbent. Third, these circles are removed from the intersection. This is the area free of the incumbent transmitter. Finally, the area within distance $d_{\text {inc }}$ from the boundary is removed. The remaining area is free of incumbent receivers. This area is seen in Fig. 3 inside the solid and rounded thick line. This is the protection zone, which is the area within which the incumbent receivers should not be subject to harmful interference caused by PS base stations. The transmission details have to be further agreed in the spectrum sharing arrangements.

\section{Spectrum sharing arrangements for combining contradicting spectrum information}

In this section, it is described how the distributed LSA controllers can use the protection zones described above for combining and verifying the spectrum information from different sources. The spectrum information sources are: the sensors, 


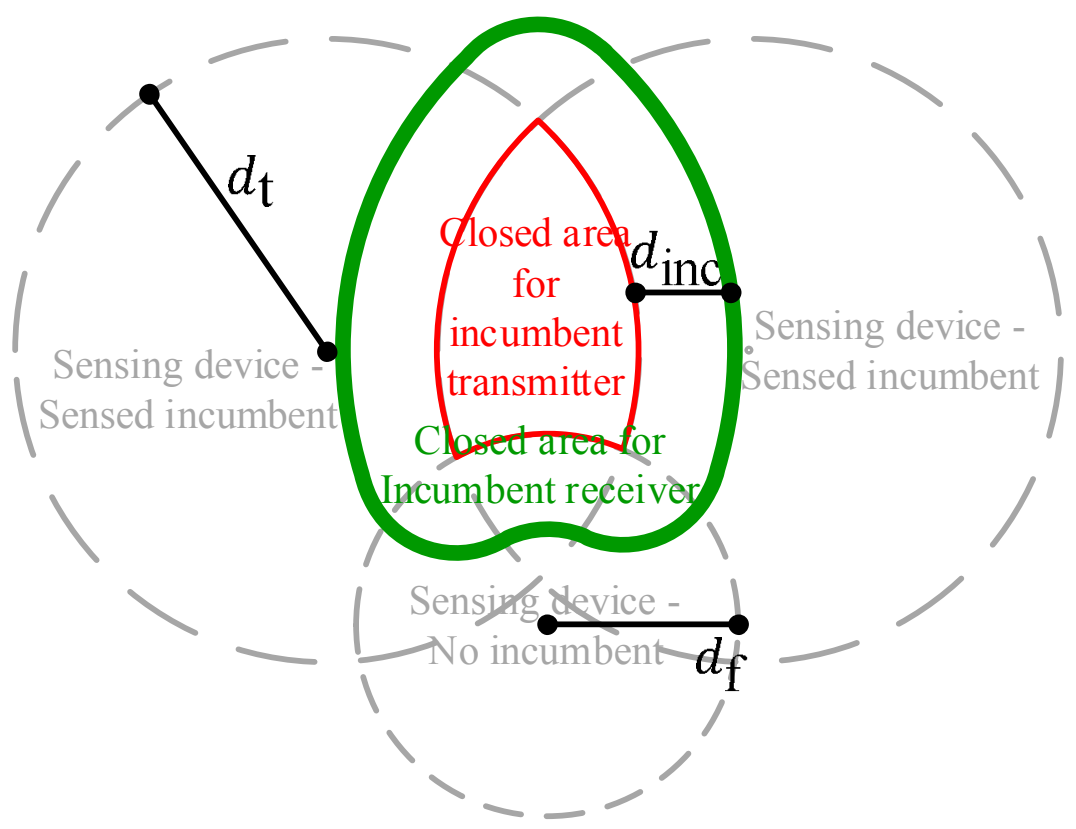

Fig. 4. Two sensors notice the same incumbent and LSA controllers calculate a closed protection zone for it, which is seen here as a green thick line. A detected transmitter is most probably closer to the sensor than $d_{\mathrm{t}}$. Moreover, a distance $d_{\mathrm{f}}$ is likely free of transmitters, given that there is no detection. 
the LSA repository, and the other LSA controllers that synchronize spectrum use of their corresponding base stations. Moreover, a lack of commercial network services can indicate that there are unused spectrum resources.

The distributed LSA controllers determine if the channel use is allowed or not and share the allowed channels between each others while selecting the channel allocations and power levels. Then, the controllers have event based listeners for noticing changes in the spectrum information and in the availability of the channels. This listener initiates a channel change for the base stations if needed.

If the channel is allowed or not depends on the LSA sharing arrangement with the incumbents. The arrangements should be agreed separately for different location types. The types of locations can for example be areas with no known commercial networks and areas with broken commercial networks. Additionally, criticality of the missions has to be taken into account in the sharing arrangements.

In a highly critical scenario, where the commercial network is down, it is straightforward for the controllers only to select the least congested LSA channel from the nearest sensor. This channel is not likely used in this particular location. Furthermore, it has the least interference.

Tables 1 and 2 are two examples of sharing arrangements for what to do with contradicting and congruent spectrum information. Here, the tables show wether the spectrum use is allowed or not by the PS base stations. Similar sharing arrangements could also be made for borderline commercial network scenarios with insufficient commercial networks.

Table 1. A sharing arrangement for LSA licensee transmissions in areas with no known commercial networks.

\begin{tabular}{|l|l|l|}
\cline { 2 - 3 } \multicolumn{1}{c|}{$\begin{array}{l}\text { Lensor(s) } \rightarrow \\
\text { LSA repository }\end{array}$} & $\begin{array}{l}\text { LSA channel } \\
\text { in this area } \\
\text { not detected free } \\
\text { available }\end{array}$ & $\begin{array}{l}\text { LSA channel } \\
\text { in this area } \\
\text { detected free }\end{array}$ \\
\hline $\begin{array}{l}\text { LSA channel } \\
\text { not available }\end{array}$ & No & Allowed \\
\hline $\begin{array}{l}\text { Old LSA information } \\
\text { with available channel }\end{array}$ & No & No \\
\hline
\end{tabular}

Note that the details of the allowed transmissions have to be further agreed. These include if the incumbent is allowed to transmit without informing the LSA repository. Moreover, the sharing arrangements resolve the transmission power and antenna height limitations, sensing methods and their detection probabilities and false and miss detection probabilities.

\section{$5 \quad$ False and miss detections}

While sensing, some amount of false and miss detections have to be tolerated. The values of these have to be agreed with the incumbent. The false detection 
Table 2. A sharing arrangement for LSA licensee transmissions in areas with broken commercial networks.

\begin{tabular}{|l|l|l|}
\cline { 2 - 3 } \multicolumn{1}{c|}{$\begin{array}{l}\text { Lensor(s) } \rightarrow \\
\text { LSA channel } \\
\text { available }\end{array}$} & $\begin{array}{l}\text { LSA channel } \\
\text { in this area } \\
\text { not detected free }\end{array}$ & $\begin{array}{l}\text { LSA channel } \\
\text { in this area } \\
\text { detected free }\end{array}$ \\
\hline $\begin{array}{l}\text { LSA channel } \\
\text { not available }\end{array}$ & Allowed & Allowed \\
\hline $\begin{array}{l}\text { Old LSA information } \\
\text { with available channel }\end{array}$ & No & Allowed \\
\hline
\end{tabular}

probability per sensor can be decided first by selecting the detection threshold correspondingly. Then, in general, the maximum miss detection probability can be made smaller by considering a smaller radius, $d_{\mathrm{f}}$, around the sensor. This radius is considered free of transmitters, given no detection. Here, the smaller detection radius $d_{\mathrm{f}}$ increases the received signal and thus increases the detection probability. Note that with a smaller $d_{\mathrm{f}}$ there might be a need for multiple sensors ${ }^{4}$ to cover the required area. Another option for covering the area are sensors that are moving for example in the air.

\section{Sensor control in practice}

The use of multiple sensors needs an efficient tool for controlling all the information. This section describes methods for the PS network to use available sensors and to notice changes in the channel information.

The information from the sensors is analyzed at the distributed LSA controllers. The sensor specific information is used by the controllers to estimate the values for $d_{\mathrm{f}}$ and $d_{\mathrm{t}}$. The sensor specific information includes at least the sensor antenna, height, location, gain and the environment of the sensor.

The PS network has a sensor manager application, that extracts the measurements of LSA licenseed channels from the available sensors. The sensor manager uses the unique commands for the corresponding sensors. Most spectrum sensors have programmable interfaces, using for example standard commands for programmable instruments (SCPI), C, or Python programming languages. The manager provides the measurements and sensor specific information for the distributed LSA controllers.

The LSA controllers access the sensing information when the PS transmissions do not disturb with sensing. For doing this, the channels are sensed before the PS base stations go on air. The information is saved for later use. However, if there are other nearby PS base stations on air, the LSA controller first waits for a communication break. Then it blocks the nearby base stations temporarily before accessing the sensed information. Moreover, the sensing can happen

\footnotetext{
${ }^{4}$ Moreover, with more detectors, false detections itself can be detected if multiple sensors give contradictory results.
} 
periodically during communication breaks. The periodical sensing is used to verify that the channel is still available. The time period should be agreed in the sharing arrangements.

\section{Conclusion}

We introduced a sensing method for PS to complement LSA spectrum information. This is done by determining reliable detection distances per sensor and by utilizing them according to the agreed sharing arrangements. A natural extension to this work is therefore to plan the required sensing network for more specific environments. The detection distances have to be further estimated for the specific sensors and for the specific incumbents.

Our scenario had a hidden assumption of simple and coarse detectors. It is also possible to consider more sophisticated sensing methods, such as power of arrival and angle of arrival techniques. These can be used for locating the detected incumbent users with a high accuracy. Then, the spectrum use could be similarly allowed given that the detected incumbents can be located to be further than their reuse distance.

\section{Acknowledgement}

The authors would like to acknowledge CORNET project consortium. This research has been financially supported by Business Finland CORNET project and by Academy of Finland 6Genesis Flagship (grant 318927).

\section{References}

1. Akyildiz, I.F., Lo, B.F., Balakrishnan, R.: Cooperative spectrum sensing in cognitive radio networks: A survey. Physical Communication 4(1), 40 - 62 (Mar 2011)

2. Benko, J.: A PHY/MAC proposal for IEEE 802.22 WRAN systems, part 1: The PHY. IEEE 802.22-06/0004r1 (Feb 2006)

3. Electron. Commun. Committee: Licensed shared access (LSA). ECC Report 205 (Feb 2014)

4. Elektrobit: Enhancing the link network performance with EB tactical wireless IP network (TAC WIN). EB Defense Newsletter (Dec 2014, Accessed Aug 2018), https://www.bittium.com/file.php?fid $=785$

5. ETSI: LTE; mission critical video over LTE. ETSI TS 122281 V15.1.0 (Jul 2018)

6. ETSI: LTE;mission critical data over LTE. ETSI TS 122282 V15.1.0 (Jul 2018)

7. ETSI: Universal mobile telecommunications system (UMTS); LTE; mission critical push to talk (MCPTT) over LTE; stage 1. ETSI TS 122179 V15.2.0 (Jul 2018)

8. ETSI: Universal mobile telecommunications system (UMTS); LTE; proximitybased services (ProSe); stage 2. ETSI TS 123303 V15.1.0 (Jul 2018)

9. ETSI: Reconfigurable radio systems (RRS); system requirements for operation of mobile broadband systems in the $2300 \mathrm{MHz}-2400 \mathrm{MHz}$ band under licensed shared access (LSA). ETSI TS 103154 V1.1.1 (Oct 2014) 
10. ETSI: Reconfigurable radio systems (RRS); system architecture and high level procedures for operation of licensed shared access (LSA) in the $2300 \mathrm{MHz}-2400$ MHz band. ETSI TS 103235 V1.1.1 (Oct 2015)

11. ETSI EN 300 392-1: Terrestrial trunked radio (TETRA); voice plus data $(\mathrm{V}+\mathrm{D})$; part 1: General network design. V1.4.1 (Jan 2009)

12. Ghasemi, A., Sousa, E.S.: Collaborative spectrum sensing for opportunistic access in fading environments. In: IEEE International Symposium on Dynamic Spectrum Access Networks. pp. 131-136 (Nov 2005)

13. Höyhtyä, M., Mämmelä, A., Eskola, M., Matinmikko, M., Kalliovaara, J., Ojaniemi, J., Suutala, J., Ekman, R., Bacchus, R., Roberson, D.: Spectrum occupancy measurements: A survey and use of interference maps. IEEE Communications Surveys Tutorials 18(4), 2386-2414 (Apr 2016)

14. Kay, S.: Fundamentals of Statistical Signal Processing: Detection Theory, vol. 2. Prentice-Hall (1998)

15. Lähetkangas, K., Saarnisaari, H., Hulkkonen, A.: Licensed shared access system possibilities for public safety. Mobile Information Systems pp. 1-12 (Jan 2016)

16. Lähetkangas, K., Saarnisaari, H., Hulkkonen, A.: Licensed shared access system development for public safety. In: Proc. European Wireless Conference. Oulu, Finland (May 2016)

17. Lehtomäki, J.: Analysis of energy based signal detection. Ph.D. dissertation, Faculty of Technology, University of Oulu, Finland (Dec 2005)

18. Mishra, S.M., Sahai, A., Brodersen, R.W.: Cooperative sensing among cognitive radios. In: IEEE International Conference on Communications. vol. 4, pp. 1658 1663 (Jun 2006)

19. Morgado, A., Gomes, A., Frascolla, V., Ntougias, K., Papadias, C., Slock, D., Avdic, E., Marchetti, N., Haziza, N., Anouar, H., Yang, Y., Pesavento, M., Khan, F., Ratnarajah, T.: Dynamic LSA for 5G networks. In: European Conference on Networks and Communications. pp. 190-194 (Jun 2015)

20. Palola, M., Hartikainen, V., Mäkeläinen, M., Kippola, T., Aho, P., Lähetkangas, K., Tudose, L., Kivinen, A., Joshi, S., Hallio, J.: The first end-to-end live trial of CBRS with carrier aggregation using $3.5 \mathrm{GHz}$ LTE equipment. In: IEEE International Symposium on Dynamic Spectrum Access Networks. pp. 1-2 (Mar 2017)

21. Sohul, M.M., Yao, M., Yang, T., Reed, J.H.: Spectrum access system for the citizen broadband radio service. IEEE Communications Magazine 53(7), 18-25 (Jul 2015)

22. Subhedar, M., Birajdar, G.: Spectrum sensing techniques in cognitive radio networks: A survey 3 (Jul 2011)

23. Uchiyama, H., Umebayashi, K., Kamiya, Y., Suzuki, Y., Fujii, T., Ono, F., Sakaguchi, K.: Study on cooperative sensing in cognitive radio based ad-hoc network. In: IEEE International Symposium on Personal, Indoor and Mobile Radio Communications. pp. 1-5 (Sep 2007)

24. Visotsky, E., Kuffner, S., Peterson, R.: On collaborative detection of tv transmissions in support of dynamic spectrum sharing. In: IEEE International Symposium on New Frontiers in Dynamic Spectrum Access Networks. pp. 338-345 (Nov 2005)

25. Wireless innovation forum: Signaling protocols and procedures for citizens broadband radio service (CBRS): Spectrum access system (SAS) - citizens broadband radio service device (CBSD) interface technical specification. WINNF-TS-0096 V 1.3.0 (Apr, 2018, Accessed Aug 2018), https://workspace.winnforum.org/higherlogic/ws/public/download/6482/

26. Yucek, T., Arslan, H.: A survey of spectrum sensing algorithms for cognitive radio applications. IEEE Communications Surveys Tutorials 11(1), 116-130 (Mar 2009) 